\title{
Wnt3a is critical for endothelial progenitor cell-mediated neural stem cell proliferation and differentiation
}

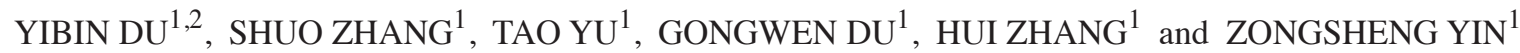 \\ ${ }^{1}$ Department of Orthopedics, First Affiliated Hospital of Anhui Medical University, Hefei, Anhui 230022; \\ ${ }^{2}$ Department of Orthopedics, Third Affiliated Hospital of Anhui Medical University, Hefei, Anhui 230061, P.R. China
}

Received July 27, 2015; Accepted July 20, 2016

DOI: $10.3892 / \mathrm{mmr} .2016 .5582$

\begin{abstract}
The present study aimed to determine whether co-culture with bone marrow-derived endothelial progenitor cells (EPCs) affects the proliferation and differentiation of spinal cord-derived neural stem cells (NSCs), and to investigate the underlying mechanism. The proliferation and differentiation of the NSCs were evaluated by an MTT cell proliferation and cytotoxicity assay, and immunofluorescence, respectively. The number of neurospheres and the number of $\beta$-tubulin III-positive cells were detected by microscopy. The wingless-type MMTV integration site family, member 3a (Wnt3a)/ $\beta$-catenin signaling pathway was analyzed by western blot analysis and reverse transcription-quantitative polymerase chain reaction to elucidate the possible mechanisms of EPC-mediated NSC proliferation and differentiation. The results revealed that co-culture with EPCs significantly induced NSC proliferation and differentiation. In addition, co-culture with EPCs markedly induced the expression levels of Wnt3a and $\beta$-catenin and inhibited the phosphorylation of glycogen synthase kinase $3 \beta$ (GSK-3 $\beta$ ). By contrast, Wnt3a knockdown using a short hairpin RNA plasmid in the EPCs reduced EPC-mediated NSC proliferation and differentiation, accompanied by inhibition of the EPC-mediated expression of $\beta$-catenin, and its phosphorylation and activation of GSK-3 $\beta$. Taken together, the findings of the present study demonstrated that Wnt3a was critical for EPC-mediated NSC proliferation and differentiation.
\end{abstract}

Correspondence to: Professor Zongsheng Yin, Department of Orthopedics, First Affiliated Hospital of Anhui Medical University, 81 Meishan Road, Hefei, Anhui 230022, P.R. China

E-mail: zongshengyin1961@163.com

Key words: neural stem cells, endothelial progenitor cells, co-culture, spinal cord injury, wingless-type MMTV integration site family, member $3 \mathrm{a}$

\section{Introduction}

Spinal cord injury causes severe neurological dysfunction, which affects patients and their families as it requires substantial long-term healthcare expenditure and leads to permanent deprivation in quality of life (1). Following spinal cord injury, neural stem cells (NSCs) at the site of damage proliferate and differentiate into several neural cell types, including neurons, astrocytes and oligodendrocytes, which are important in cell-replacement therapy for neurological dysfunction (2). NSCs are considered to be a potential cell therapy for reconstruction and regeneration of the brain and spinal cord following injury (3). The transplantation of NSCs into the injured sites can potentially replace lost cells and become involved in anatomical regeneration $(4,5)$. However, which of the cell types the NSCs differentiate into, and the proportion, determines patient prognosis, and the poor proliferation rate of NSCs has limited the practical use of NSC-based therapy (4).

There is increasing evidence that endothelial progenitor cells (EPCs) contribute to angiogenesis by promoting migration and proliferation (6). In addition, EPCs contribute directly and indirectly to neovascularization, and are incorporated into injured vessels to become mature endothelial cells in response to tissue injury, which improves clinical outcomes in patients with ischemic disease $(6,7)$. It has been shown that patients with spinal cord injury have high levels of circulating bone marrow-derived EPCs, and chemoattractive and proangiogenic cytokines in their blood within the first day of illness (8). A close association between NSCs and vascular cells in the adult central nervous system has been shown in the 'vascular niche' (9). A previous study demonstrated that the transplantation of EPCs promotes astrogliosis and functional recovery following spinal cord injury (10). However, the behavior of EPCs on NSCs and their underlying mechanism remain to be fully elucidated.

The present study aimed to determine whether co-culture with bone marrow-derived EPCs affects spinal cord-derived NSC proliferation and differentiation. The data obtained in the present study is the first, to the best of our knowledge, to demonstrate that co-culture with bone marrow-derived EPCs promoted the proliferation and differentiation of spinal cord-derived NSC, at least in part, via modulation of the wingless-type MMTV integration site family, member 
3a (Wnt3a)/ $\beta$-catenin signaling pathway. In conclusion, the results provided novel molecular insight into EPC-mediated neurogenesis during the repair of spinal cord injury.

\section{Materials and methods}

Animals. All animal procedures were approved by the Animal Ethics Committee of the Institutional Animal Care and Use Committee of Anhui Medical University (Hefei, China), in accordance with the Guide for the Care and Use of Laboratory Animals in China (11). Bone marrow progenitor cells were harvested from male Sprague-Dawley (SD) rats (90-120 g; $\mathrm{n}=25$ ). NPCs were harvested from newborn SD rats (5-7 days old; $n=60$ ). They were kept in a specific pathogen free environment at $22 \pm 1^{\circ} \mathrm{C}$ under a $14 / 10 \mathrm{~h}$ light-dark cycle with free access to food and access to water under controlled environmental conditions.

Materials. Endothelial basal medium (EBM)-2 and EGM-2 Single Quots, containing $10 \mathrm{ml}$ fetal bovine serum (FBS), $0.2 \mathrm{ml}$ hydrocortisone, $2 \mathrm{ml}$ human fibroblast growth factor (hFGF)-B, $0.5 \mathrm{ml}$ vascular endothelial growth factor (VEGF), $0.5 \mathrm{ml}$ R3-IGF-1, $0.5 \mathrm{ml}$ ascorbic acid, $0.5 \mathrm{ml} \mathrm{hEGF,}$ $0.5 \mathrm{ml} \mathrm{GA}-1000$ and $0.5 \mathrm{ml}$ heparin, were purchased from Clonetics (San Diego, CA, USA). Dulbecco's modified Eagle's medium/F12 (DMEM/F12) medium,FBS, B27 supplement and L-glutamic acid (L-glutamine) were purchased from Gibco; Thermo Fisher Scientific, Inc. (Waltham, MA, USA). Basic (b) FGF and epidermal growth factor (EGF) were purchased from Peprotech, Inc. (Rocky Hill, NJ, USA). The rat bone marrow lymphocyte isolation kit was purchased from Tianjin Hao Yang Biological Products Technology Co., Ltd. (Tianjin, China). Rat fibronectin was purchased from Gene Operation, Inc. (Ann Arbor, MI, USA); DiI-labeled acetylated low-density lipoprotein (Di1-Ac-LDL) and Lipofectamine 2000 were purchased from Invitrogen; Thermo Fisher Scientific, Inc. Fluorescein isothiocyanate UEA-1 (FITC-UEA-1), and poly-lysine were purchased from Sigma-Aldrich; Thermo Fisher Scientific, Inc. Antibodies against VEGF receptor (VEGFR)-2, $\beta$-tubulin III, $\beta$-actin and nestin were purchased from Santa Cruz Cruz Biotechnology, Inc. (Santa Cruz, CA, USA). Antibodies against Wnt3a, phosphorylated (p)-glycogen synthase kinase $3 \beta$ (GSK-3 $\beta$ ), $p$ - $\beta$-catenin, GSK-3 $\beta$ and $\beta$-catenin were purchased from Cell Signaling Technology, Inc. (Danvers, MA, USA). Antibody against CD133 was purchased from Biorbyt, Ltd. (San Fransisco, CA, USA). Rabbit anti-glial fibrillary acidic protein antibodies were purchased from Abcam (Cambridge, UK). The recombinant plasmid, pEGFP-short hairpin (sh) RNA-wnt3a, was purchased from Hefei Hao Xiang Biological Technology Co., Ltd (Anhui, China).

Isolation and culture of bone marrow-derived EPCs. The SD rats (90-120 g) were sacrificed with an excess of $10 \%$ chloral hydrate anesthesia, following which both femurs and tibias were surgically dissected. The bone marrow mononuclear cell population was isolated using a commercially available kit (R\&D systems, Inc., Minneapolis, MN, USA), according to the manufacturer's protocol. The bone marrow mononuclear cells were then re-suspended in EBM-2 complete medium. To isolate the EPCs, the bone marrow mononuclear cells $\left(5 \times 10^{5}\right.$ cells/well) were plated on bovine fibronectin-coated 24-well plates. The plates were incubated in $5 \% \mathrm{CO}_{2}$ at $37^{\circ} \mathrm{C}$. The medium was replaced every 3 days until the first passage cells were $\sim 70 \%$ confluent (14 days). The EPCs were identified by the expression of cell surface markers, CD133 and VEGFR-2, using fluorescence microscopy. In addition, the uptake of fluorescent Dil-ac-LDL was evaluated using confocal microscopy. The binding of UEA-1 was determined using FITC-conjugated UEA-1.

Isolation and culture of spinal cord-derived NSCs. Newborn SD rats (5-7 days old) were sacrificed by cervical dislocation. The thoracolumbar spinal cord, stripped of soft meninges and blood vessels, were placed in ice-cold DMEM/F12 for further dissection. The spinal cord was cut it into sections measuring $1 \mathrm{~mm}^{3}$ with ophthalmic scissors, and filtrated through a 200 mesh cell sieve following repeated pipetting turbid suspension. The dissociated cell suspension was centrifuged at $800 \mathrm{xg}$ for $5 \mathrm{~min}$ at room temperature, and the pellet was seeded $\left(1 \times 10^{6}\right.$ cells $\left./ \mathrm{ml}\right)$ into flasks containing DMEM/F-12 with $2 \%$ B27, $20 \mathrm{ng} / \mathrm{ml}$ EGF, $10 \mathrm{ng} / \mathrm{ml} \mathrm{bFGF}$ and $0.6 \mathrm{mg} / \mathrm{ml} \mathrm{L}$-glutamine. The flasks were incubated in $5 \% \mathrm{CO}_{2}$ at $37^{\circ} \mathrm{C}$. After $48 \mathrm{~h}$, the medium was replaced the remove the non-adherent cells, and was replaced every 3 days thereafter. All the cells used in the experiments were obtained from passages 3-10. The NSCs were identified by positive staining for nestin under a light microscope (Carl Zeiss Inc., Jena, Germany), a molecular marker for multipotent NSCs (12), which is required for the proliferation and self-renewal of NSCs.

Plasmid transfection. The EPCs were transfected with the mouse wnt3a pEGFP-shRNA plasmid using lipofectamine 2000 and Opti-MEM medium (Invitrogen; Thermo Fisher Scientific, Inc.). Briefly, the EPCs were plated on 6-well plates at $70-80 \%$ confluence $24 \mathrm{~h}$ prior to transfection. The Wnt3a pEGFP-shRNA plasmid $(2 \mu \mathrm{g})$, Lipofectamine $2000(2 \mu \mathrm{l})$ and Opti-MEM were mixed and incubated at room temperature for $5 \mathrm{~min}$. The plasmid-oligofectamine complexes were added to the cells for $24 \mathrm{~h}$ and the medium was replaced with fresh serum-free EGM-2 following transfection for $72 \mathrm{~h}$. Transfection efficiency was determined by the percentage of GFP-positive cells. Knockdown of wnt3a was assessed using western blot analysis.

Immunofluorescence assessment. For the in vitro experiments, the EPCs or NSCs were grown on glass slides in 6-well plates. The cells were fixed in $4 \%$ paraformaldehyde for $30 \mathrm{~min}$ at room temperature. Immunostaining was performed using mouse monoclonal anti-VEGR2 (1:150; Biorbyt, Ltd.), polyclonal rabbit anti-CD133 (1:150) and/or polyclonal rabbit anti-nestin (1:150; Sigma-Aldrich; Thermo Fisher Scientific, Inc.) and FITC-conjugated anti-rabbit or anti-mouse $\operatorname{IgG}(1: 200$; Sigma-Aldrich; Thermo Fisher Scientific, Inc.) and tetra methyl rhodamyne iso-thiocyanate anti-rabbit or mouse $\operatorname{IgG}(1: 200$; Sigma-Aldrich; Thermo Fisher Scientific, Inc.) secondary antibodies were utilized, and counterstaining for nuclei was performed using 2-(4-Amidinophenyl)-6-indolecarbamidine dihydrochloride. Immunofluorescence was visualized using a fluorescent microscope (Olympus Corporation, Tokyo, Japan). The results were based on three independent analyses. 
To determine the uptake of Dil-Ac-LDL and binding of FITC-UEA-1, the EPCs were incubated with Dil-Ac-LDL overnight at $37^{\circ} \mathrm{C}$, and fixed with $4 \%$ paraformaldehyde for $20 \mathrm{~min}$. The cells were then incubated with FITC-UEA-1 for $1 \mathrm{~h}$ at $37^{\circ} \mathrm{C}$, and examined under a fluorescent microscope (Olympus Corporation).

EPC/NSC co-culture assay. To investigate the effect of EPC co-culture on the differentiation and proliferation of NSCs, an EPC/NSC co-culture assay was performed, as described in a previous report (13) with minor modifications. The EPCs and NSCs were separately seeded into 24-well (2x10 ${ }^{5} \mathrm{NSCs} /$ well; $1 \times 10^{5} \mathrm{EPCs} /$ insert $)$ or 6 -well $\left(6 \times 10^{5} \mathrm{NSCs} /\right.$ well; $5 \times 10^{5} \mathrm{EPC} /$ insert $)$ Transwell plates (0.4 $\mu \mathrm{m}$ pore-size; Corning Costar, St Louis, MO, USA). The co-culture system was maintained in culture medium with DMEM/F12+ serum-free EBM-2 (1:1) in $5 \% \mathrm{CO}_{2}$ at $37^{\circ} \mathrm{C}$. Following co-culture for 7 days, the cells obtained from the differentiated NSCs were fixed and labeled with the neuronal specific marker, $\beta$-tubulin III (14), and neuronal proteins were extracted for western blot analysis. Neuronal viability was measured using a 3-(4,5-dimethyl-thiazol-2-yl)-2,5-diphenyl-tetrazolium bromide (MTT) assay.

Cell proliferation assay. Following co-culture with EPCs for different periods of time, the viability of the NSCs was determined using an MTT Cell Proliferation and Cytotoxicity Assay kit (Beyotime Institute of Biotechnology, Haimen, China), according to the manufacturer's protocol. The cell viability was measured at $490 \mathrm{~nm}$ with a microplate reader (Tecan M200; Tecan Austria GmbH, Salzburg, Austria). NSC proliferation was measured using a BrdU cell proliferation kit (Roche, Mannheim, Germany), according to the manufacturer's protocol. The cell proliferation was measured at $450 \mathrm{~nm}$ with a microplate reader (Tecan M200; Tecan Austria GmbH).

Measurement of the numbers of neurospheres. In order to investigate the proliferation potential of the NSCs in the presence and absence of EPC co-culture, a neurosphere growth kinetics assay was performed, as described earlier. The NSC culture was passaged by gentle trituration, and the resulting single-cell suspension of NSCs was replated in a 12-well plate at a density of $5 \times 10^{4}$ cells/well for co-culture with or without EPCs. The number and diameter of the neurospheres were measured in all groups using an inverted phase-contrast microscope (Leica, Mannheim, Germany) and analyzed using Image J software (v1.50a, National Institutes of Health, Bethesda, MA, USA).

Reverse transcription-quantitative polymerase chain reaction $(R T-q P C R)$ analysis. Total RNA was extracted from the differentiated NSCs using TRIzol (Takara Biotechnology Co., Ltd., Dalian, China). The total RNA was isolated and purified using an RNeasy minikit (Takara Biotechnology Co., Ltd.) with the addition of RNase-free DNase I (Takara Biotechnology Co., Ltd.). The total RNA (1 $\mu \mathrm{g})$ was reverse transcribed using a one-step RT kit (Takara Biotechnology Co., Ltd.), and the resulting complementary DNA was used as a PCR template for determining the messenger RNA (mRNA) expression levels using a SYBR-Green
Quantitative PCR kit (Takara Biotechnology Co., Ltd.) with the iCycler iQ system (Bio-Rad Laboratories, Inc., Hercules, CA, USA). Glyceraldehyde-3-phosphate dehydrogenase (GAPDH) was used as the housekeeping gene. Relative expression was calculated using the $\Delta \Delta \mathrm{Cq}$ method (15). Quantification was performed using standard curves derived from the expression of the gene relative to that of GAPDH. The rat-specific primers for Wnt- $3 \alpha, \beta$-catenin and GAPDH were as follows (16): Wnt-3 $\alpha$, forward 5'-GCTACTCGGCCT CCTGCT-3' and reverse 5'-GGCCAGAGACGTGTACTG CT-3'; $\beta$-catenin, forward 5'-GACCACAAGCAGAGTGCT GA-3' and reverse 5'-ACTCGGGTCTGTCAGGTGAG-3'; GAPDH, forward 5'-AGGTTGTCTCCTGCGACTTCA and reverse 5'-TGGTCCAGGGTCCAGGGTTTCTTACTCC-3'.

Western blot analysis. Western blot analysis was performed, as previously described (17). Total lysis of the cells was conducted with RIPA buffers (Thermo Fisher Scientific, Inc.) and protein concentration was determined with a bicichoninic acid protein assay kit (Thermo Fisher Scientific, Inc.). Equal quantities $(50 \mu \mathrm{g})$ of proteins were separated and transferred onto polyvinylidine difluoride membranes. The membranes were blocked with $5 \%$ non-fat dried milk, following which the membranes were probed overnight at $4{ }^{\circ} \mathrm{C}$ with the following antibodies: Rabbit monoclonal anti-Wnt3a (Ab2721 1:1,000), rabbit monoclonal anti-p-GSK-3 $\beta$ (Ab5558; 1:2,000), rabbit monoclonal anti-p- $\beta$-catenin (Ab9561; 1:2,000), rabbit monoclonal anti-GSK-3 $\beta$ (Ab12456; 1:2,000), rabbit monoclonal anti- $\beta$-catenin (Ab4176; 1:2,000) or mouse monoclonal anti- $\beta$-actin (Ab3700; 1:2,000) (all from Abcam, Cambridge, MA, USA). This was followed by incubation with either horseradish peroxidase-conjugated goat anti-rabbit (ZB-2301) or anti-mouse antibody(ZB-2305) (1:5,000; Zhongshan Golden Bridge Biotechnology, Beijing, China) for $2 \mathrm{~h}$ at room temperature. Immunoreactive proteins were visualized using enhanced chemiluminescence, and signal intensity was detected and quantified using Alpha Imager (Alpha Innotech Corporation, San Leandro, CA, USA).

Enzyme-linked immunosorbent (ELISA) assay. The level of VEGF in the medium of the EPCs was measured using a commercially available ELISA kit (R\&D Systems Europe, Ltd., Abingdon, UK), according to the manufacturer's protocol. After 1, 3, 5, 7 and 14 days of culture, the cell supernatants were collected to measure the levels of VEGF in the medium. Each assay was repeated at least three times.

Statistical analysis. The results of the experimental investigations are expressed as the mean \pm standard error of the mean. Differences between the mean values of multiple groups were analyzed using one-way analysis of variance with Tukey's test for post-hoc comparisons. All data analysis was performed with the use of GraphPad Prism 5 software (GraphPad Software, Inc., San Diego, CA, USA). P<0.05 was considered to indicate a statistically significant difference.

\section{Results}

Isolation and culture of bone marrow-derived EPCs and spinal cord-derived NSCs. Bone marrow mononuclear 
A

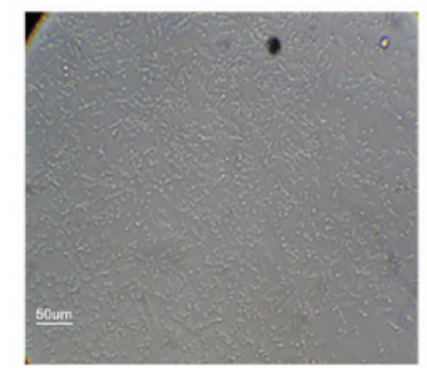

D

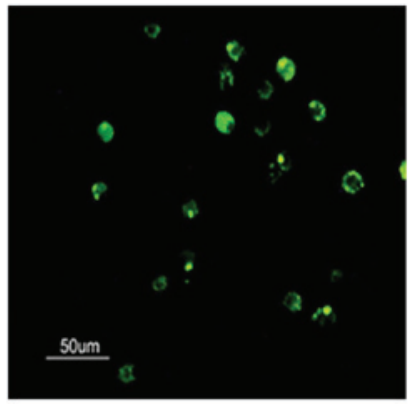

G

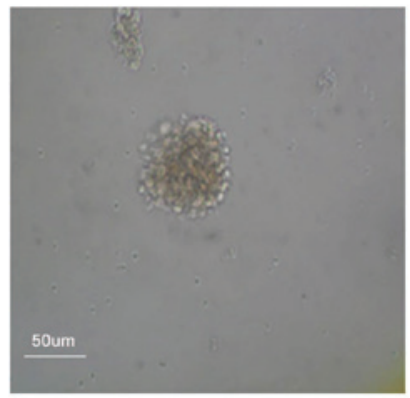

B

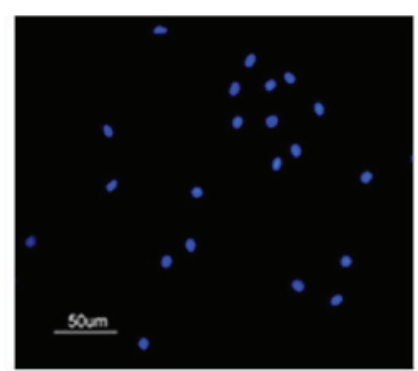

$\mathbf{E}$

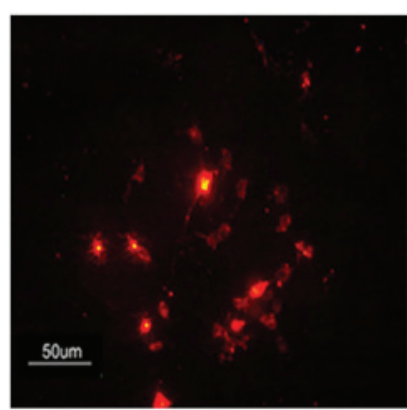

H

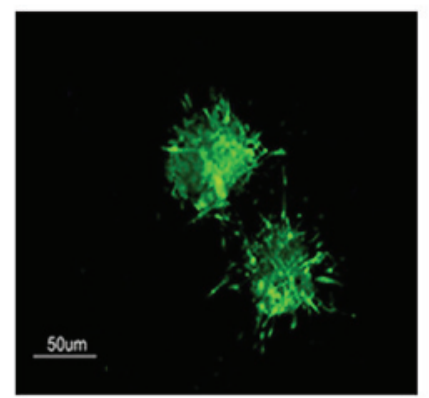

C

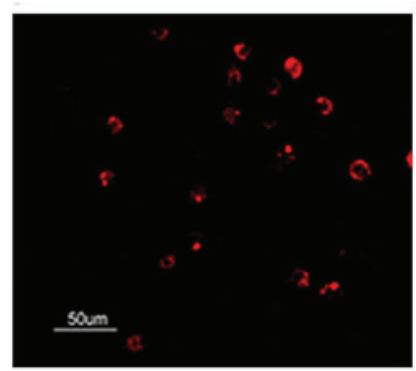

F

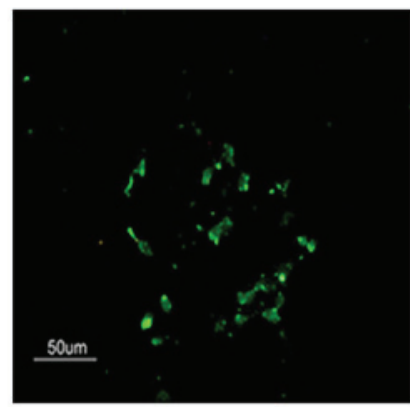

I

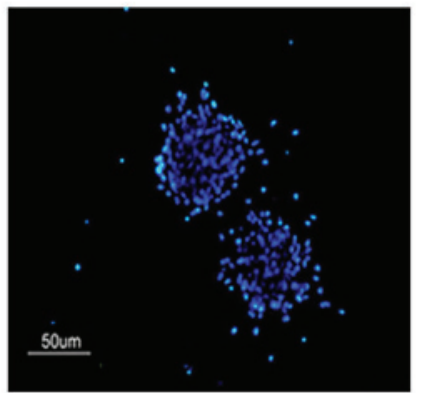

Figure 1. Isolation and culture of bone marrow-derived EPCs and spinal cord-derived NSCs. Images show bone marrow-derived EPCs (A) 14 days following isolation and (B) following DAPI staining. The (C) expression of vascular endothelial growth factor-2 and (D) CD133, the (E) uptake of DiI-labeled acetylated low-density lipoprotein and (F) binding to fluorescein isothiocyanate UEA-1 were analyzed. (G) Spinal cord-derived NSCs 21 days following isolation, (H) expressing nestin and (I) following DAPI staining. Scale bar=50 $\mu \mathrm{m}$. EPCs, endothelial progenitor cells; NSCs, neural stem cells; DAPI, 2-(4-Amidinophenyl)-6-indolecarbamidine dihydrochloride.

cells were isolated by density gradient centrifugation; the isolated mononuclear cells were small and round in shape. After 24 h, a small number of adherent cells appeared. After 7 days, a proportion of the mononuclear cells had become spindle-shaped. On day 14, the nearby colonies had fused with each other, exhibiting a larger cell monolayer with a cobblestone-like morphology (Fig. 1A). Certain EPCs formed linear cord-like structures during cultivation, which is consistent with a previous report (18). The EPCs were identified by positive staining for VEGFR-2, an endothelial cell surface marker (Fig. 1C) and CD133, a progenitor cell surface antigen (Fig. 1D) (19). The EPCs are further identified by their ability to take up Dil-Ac-LDL and bind FITC-UEA-1 (Fig. 1E and F).

A specific characteristic of spinal cord-derived NSCs is the ability to self-renew. The ability to passage neurospheres clonally is an indicator of self-renewal. In the present study, only individual cells were observed 1 day post-seeding, with no cell spheres observed. The assembly of the neurospheres was slow and the spheres were relatively small. After 7-21 days of subculture, phase contrast microscopy indicated that neurosphere formation had occurred (Fig. 1G). The neurospheres of the NSCs were identified by positive staining for nestin (Fig. 1H).

Co-culture with EPCs promotes the proliferation and differentiation of NPCs. To assess the effects of co-culture with EPCs on the proliferation and viability of the NSCs, the NSCs were co-cultured with EPCs for different time periods and their viability was measured using an MTT assay. As shown in Fig. 2A, co-culture with EPCs significantly increased the proliferation of the NSCs in a time-dependent manner. The effects of co-culture with EPCs on NSC proliferation were determined using a BrdU incorporation assay and neurosphere growth kinetic assay. Following co-culture with EPCs for 7 days, the number of neurospheres was significantly increased (Fig. 2B). The co-culture with EPCs was accompanied by a significant increase in the number of $\mathrm{BrdU}^{+}$cells, also in a time-dependent manner (Fig. 2C). In addition, co-culture with EPCs for 7 days significantly induced differentiation, as evidenced by the increase of $\beta$-tubulin III-positive cells (Fig. 2D). These results suggested that co-culture with EPCs significantly induced NSC proliferation and differentiation. 
A

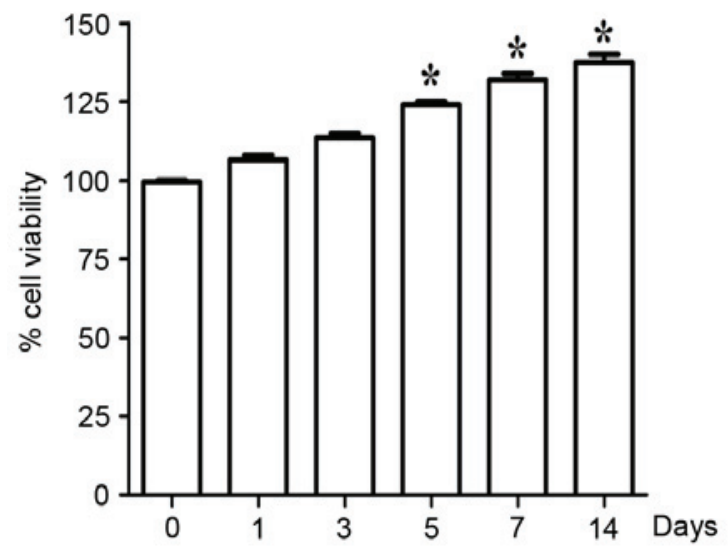

C

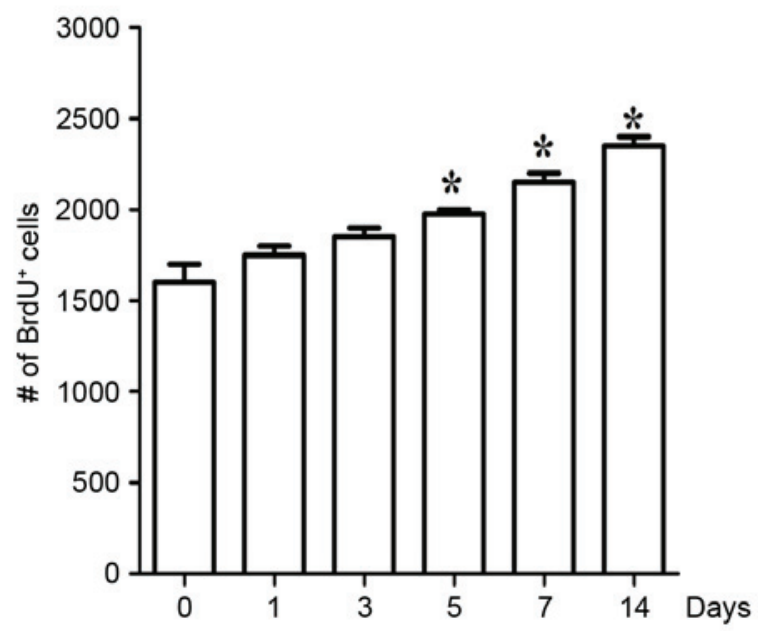

B
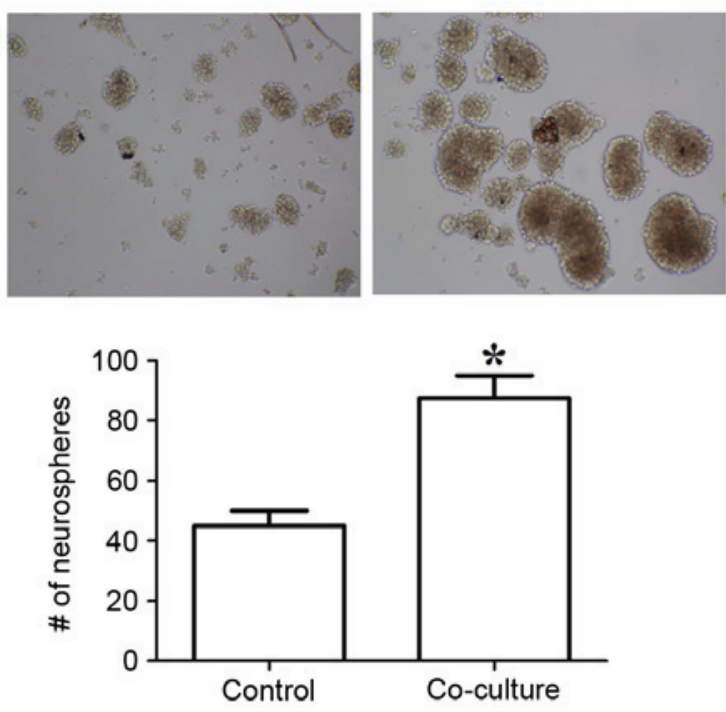

D
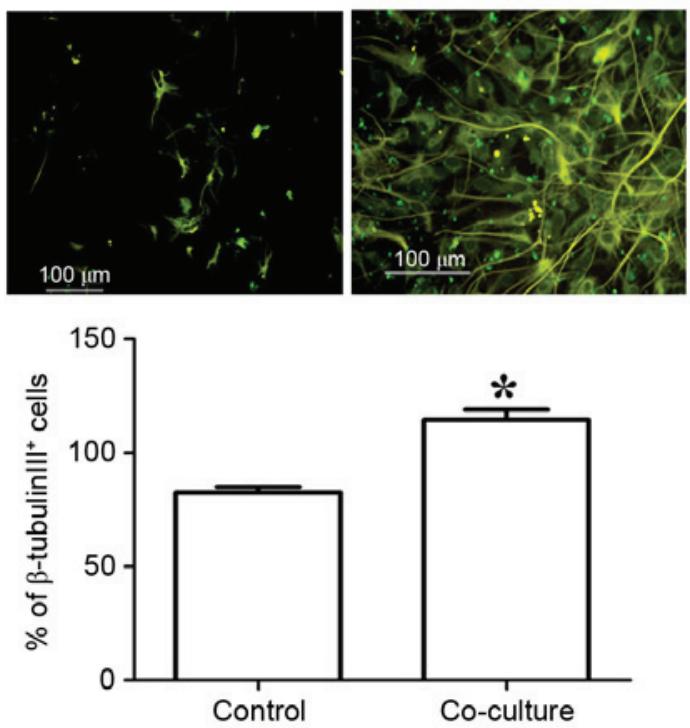

Figure 2. Co-culture with EPCs promotes NSC proliferation and differentiation. (A) NSCs were seeded into 24-well plates and co-cultured with or without EPCs for the indicated time periods. (A) Cell viability of the NSCs was assessed using a 3-(4,5-dimethyl-thiazol-2-yl)-2,5-diphenyl-tetrazolium bromide assay. Values are expressed as the mean \pm standard error of the mean $(n=3)$. (B) Images showing the number of neurospheres (magnification, $x 100)$. (C) Cell proliferation of the NSCs was analyzed using a BrdU ELISA assay. (D) Images show NSC differentiation ( $\beta$-tubulin III-positive cells). Values are expressed as the mean \pm standard error of the mean $(\mathrm{n}=3)$. Scale bar=100 $\mu \mathrm{m}$. "P $<0.05$, vs. NSCs alone. EPCs, endothelial progenitor cells; NSCs, neural stem cells.

Co-culture with EPCs induces activation of the Wnt3a/ $\beta$-catenin pathway in NSCs. Previous studies have identified that the $\mathrm{Wnt} / \beta$-catenin pathway may be critical in the regulation of NSC proliferation and differentiation $(20,21)$. Therefore, the present study examined the effects of co-culture with EPCs on the gene and protein expression levels of the Wnt/ $\beta$-catenin pathway in NSCs. The results of the RT-qPCR analysis showed that co-culture with EPCs significantly enhanced the gene expression levels of $\beta$-catenin and Wnt3a in the NSCs (Fig. 3A and B). The results of the western blot analysis further confirmed that co-culture with EPCs significantly increased the expression levels of $\beta$-catenin and Wnt3a (Fig. 3C and D). Of note, co-culture with EPCs also significantly reduced the phosphorylation of GSK-3 $\beta$ (Fig. 3E). Thus, co-culture with EPCs activated the $\mathrm{Wnt} / \beta$-catenin pathway and inhibited the activation of GSK-3 $\beta$, which contributed to the promotion of NSC proliferation and differentiation.
Wnt3a knockdown inhibits EPC co-culture-mediated NSC proliferation and differentiation. NSCs are functionally characterized as cells with the capacity to proliferate, self-renew and produce populous progeny, which can differentiate into neurons, astrocytes and oligodendrocytes (22-24). To determine whether Wnt3a is essential in EPC co-culture-mediated NSC proliferation and differentiation, genetic inactivation was performed via the transfection of EPCs with Wnt3a shRNA. As shown in Fig. 4A, transfection with the Wnt3a shRNA plasmid markedly reduced the expression of Wnt3a in the EPCs $(\mathrm{P}<0.05)$. EPCs are one of source of angiogenic mediators (25). To examine the effect of Wnt3a knockdown on the secretory function of EPCs, the levels of VEGF were examined in the EPCs following Wnt3a knockdown. The results showed that Wnt3a knockdown had no effect on the production of VEGF in the EPCs following culture for different time periods (Fig. 4B). However, Wnt3a knockdown in the EPCs significantly reduced EPC-mediated NSC 


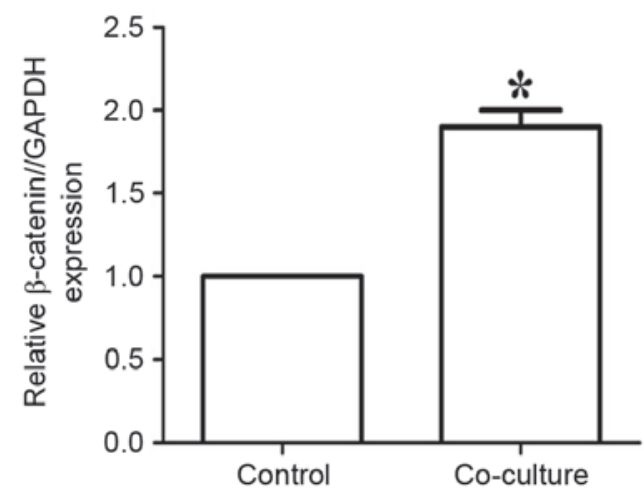

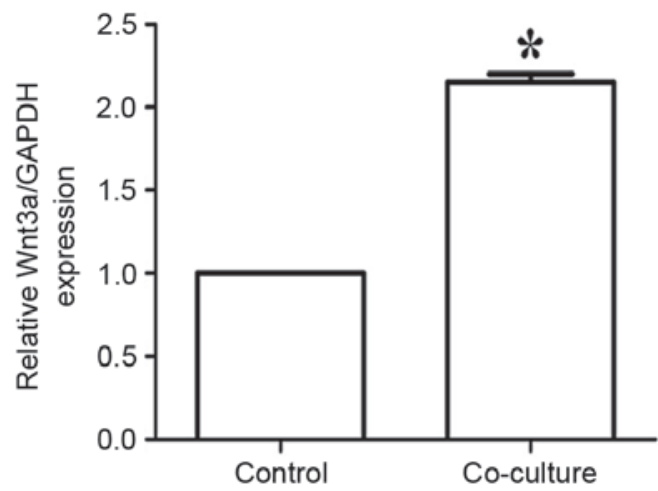

C
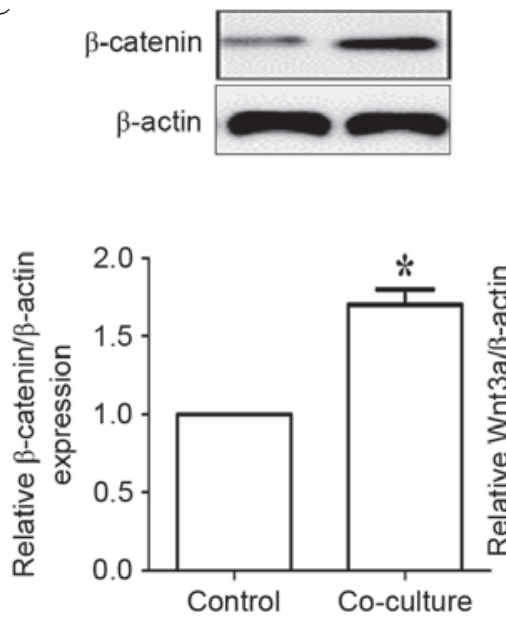

D

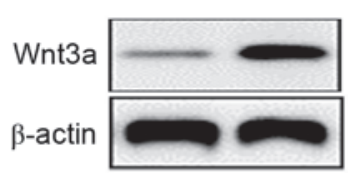

$\mathbf{E}$
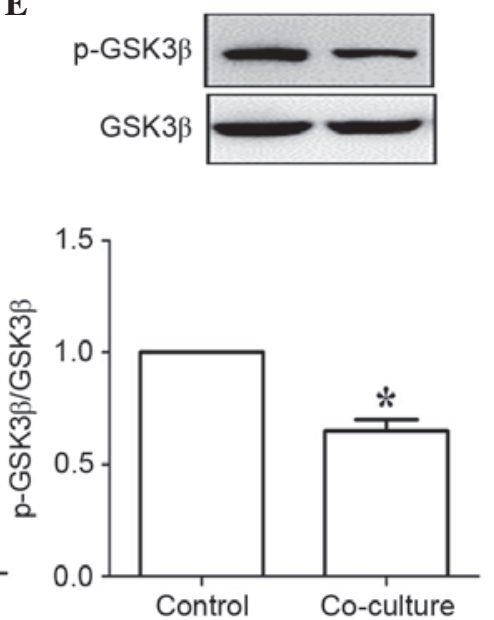

Figure 3. Co-culture with EPCs induces activation of the Wnt3a/ $\beta$-catenin pathway in NSCs. Following co-culture for 7 days, reverse transcription-quantitative polymerase chain reaction analysis was used to analyze the mRNA levels of (A) $\beta$-catenin and (B) Wnt3a. GAPDH was used as the housekeeping gene. Western blot analysis was used to determine the protein levels of (C) $\beta$-catenin, (D) Wnt3a and (E) p-GSK3 $\beta$. $\beta$-actin was used as a loading control. Values are expressed as the mean \pm standard error of the mean $(n=3)$. ${ }^{*} \mathrm{P}<0.05$, vs. NSCs alone. EPCs, endothelial progenitor cells; NSCs, neural stem cells; Wnt3a, wingless-type MMTV integration site family, member 3a; p-GSK3 $\beta$, phosphorylated glycogen synthase kinase $3 \beta$.

proliferation, as demonstrated by the BrdU incorporation assay, following co-culture for 7 days (Fig. 4C). Similarly, Wnt3a knockdown in the EPCs significantly decreased the number of NSC neurospheres in the co-culture system, detected using a neurosphere growth kinetic assay (Fig. 4D). In addition, following Wnt3a knockdown in the EPCs, $\beta$-tubulin III-positive staining was reduced in the co-culture system (Fig. 4E). Collectively, these results suggested that Wnt3a is critical for EPC-mediated NSC proliferation and differentiation in this co-culture system.

Wnt3a knockdown inhibits the activation of $\beta$-catenin signaling in the co-culture system. To further assess the function of Wnt3a signaling, the NSCs were co-cultured with EPCs with Wnt3a knockdown. As shown in Fig. 5A and B, the cellular gene and protein levels of $\beta$-catenin were significantly increased in the NSCs co-cultured with EPCs for 7 days $(\mathrm{P}<0.05)$, and were significantly decreased in the co-culture system containing EPCs with Wnt3a knockdown. Wnt3a knockdown in the EPCs also markedly reduced the phosphorylation and nuclear translocation of $\beta$-catenin (Fig. 5C and D). Wnt3a knockdown in the EPCs markedly enhanced the phosphorylation of GSK-3 $\beta$ following co-culture for 7 days
(Fig. 5E). These results demonstrated that Wnt $3 \mathrm{a} / \beta$-catenin signaling was responsible for the EPC-mediated NSC proliferation and differentiation.

\section{Discussion}

The present study demonstrated that co-culture with EPCs promoted spinal cord-derived NSC proliferation and differentiation, and that these effects were not observed following Wnt3a knockdown in the EPCs. The data further demonstrated that Wnt3a was critical for EPC-mediated NSC proliferation and differentiation through modulation of the $\beta$-catenin and GSK-3 $\beta$ signaling pathway.

Spinal cord injury is a serious threat to human health and quality of life. One option for treating spinal tissue damage includes the replacement of lost neuronal cells, and NSC transplantation therapy offers the potential to promote the repair of neuronal loss and functional recovery of spinal cord-transected rats $(26,27)$, as they have the capacity to differentiate into neurons and oligodendrocytes. However, the survival of transplanted NSCs is the primary problem associated with this therapy. The microenvironment may also affect the mechanism by which transplanted NSCs induce proliferation and 
A
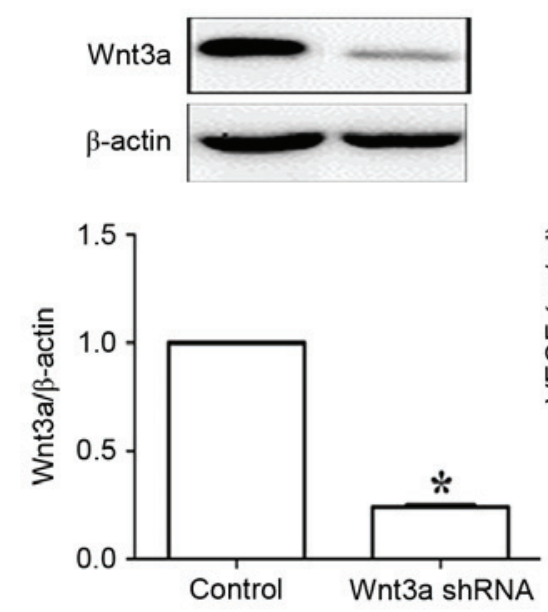

D
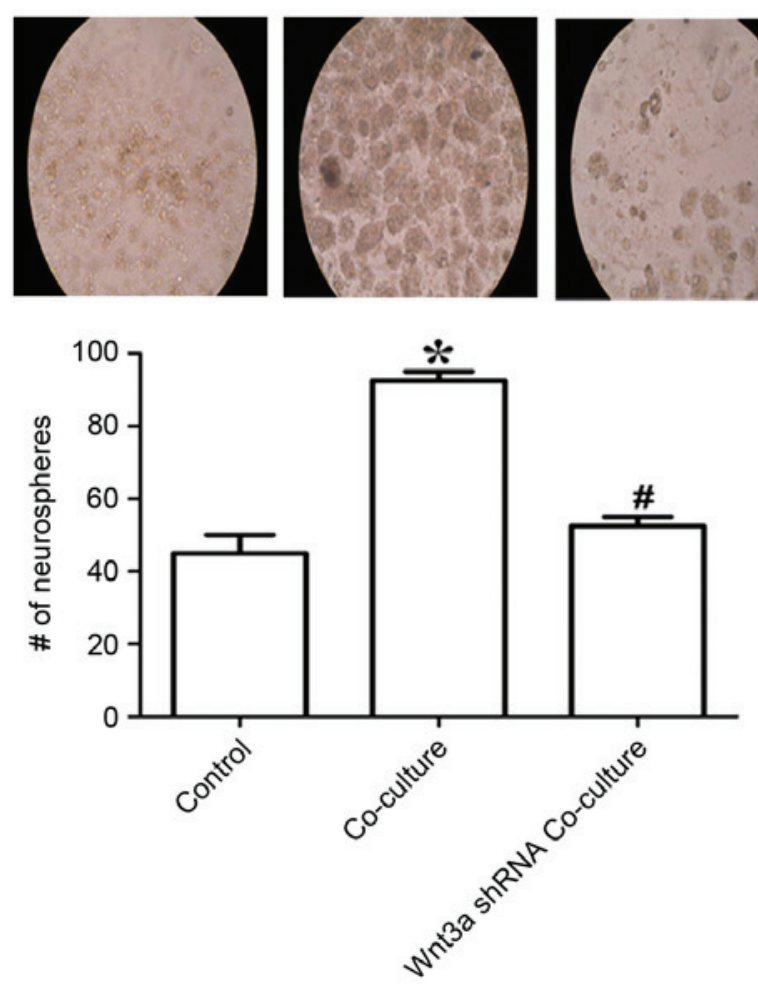

\section{B}

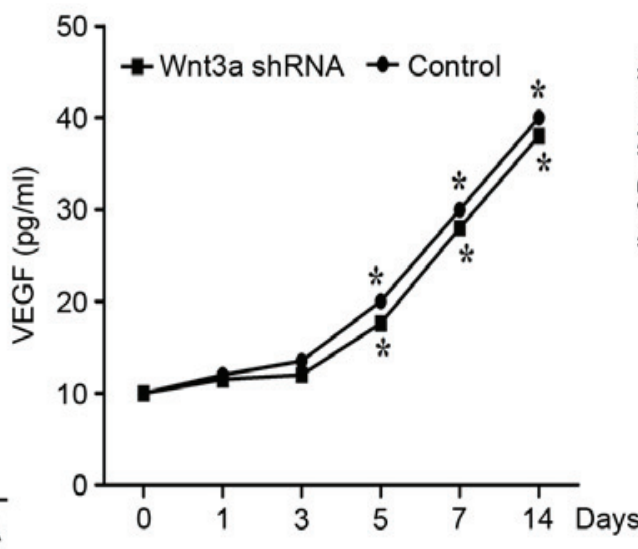

E
C

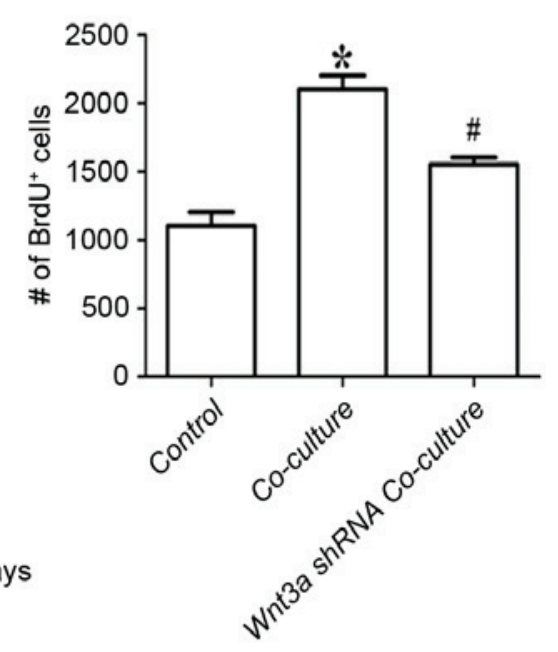

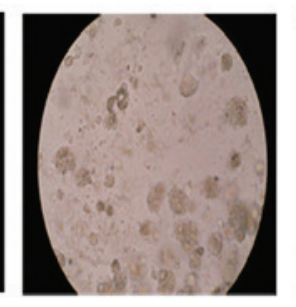
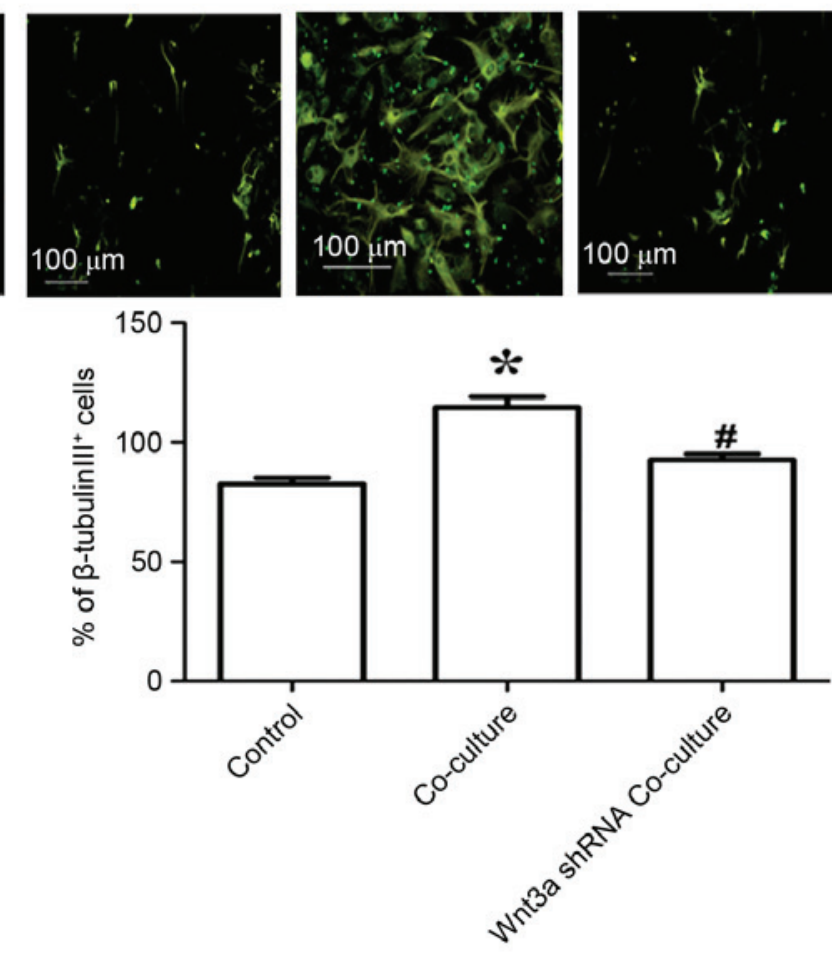

Figure 4. Wnt3a knockdown inhibits EPC co-culture-mediated NSC proliferation and differentiation. (A) Following treatment with a Wnt3a shRNA plasmid, western blot and densitometric analyses were performed to determine the level of Wnt3a, $\beta$-actin was used as loading control. (B) Following Wnt3a knockdown for the indicated time periods, the levels of VEGF released in the EPCs were determined. After 7 days co-culture with the EPCs, the (C) proliferation of the NSCs, the (D) number of neurospheres (magnification, x100) and (E) differentiation of NSCs were determined. Scale bar=100 $\mu \mathrm{m}$. Values are expressed as the mean \pm standard error of the mean $(n=3)$. ${ }^{*} \mathrm{P}<0.05$, vs. NSCs alone; ${ }^{\#} \mathrm{P}<0.05$, vs. EPC co-culture. EPCs, endothelial progenitor cells; NSCs, neural stem cells; Wnt3a, wingless-type MMTV integration site family, member 3a; shRNA, short hairpin RNA.

differentiation to promote repair following spinal cord injury. Previous studies have shown that co-culture with certain cells, including glial cells, olfactory ensheathing cells (28) and mesenchymal stromal cells (29) can promote the neurogenesis of NSCs. Vascular endothelial cells also promote NSC self-renewal and neurogenesis (30). Endothelial cells secrete numerous factors, several of which have been implicated in regulating the germinal niche (31). In the present study, co-culture with EPCs was found to significantly induce cell proliferation and the expression of nestin in the neurospheres, suggesting that co-culture with EPCs may have an effect on the microenvironment and enhance the proliferation of NSCs.
In addition, the co-culture with EPCs markedly induced the differentiation of NSCs, which was consistent with previous reports that endothelial cells induce the differentiation of NOSCs into neurons and astrocytes $(32,33)$. Taken together, the results of the present study indicated that co-culture with EPCs may stimulate NSC proliferation and differentiation via cell-cell communication.

The activation of $\mathrm{Wnt} / \beta$-catenin signaling is a key factor in initiating and promoting neurogenesis due to its ability to selectively trigger the expression of a panel of neuronal-associated genes for NSC proliferation and differentiation (34). However, inhibition of the $\mathrm{Wnt} / \beta$-catenin 
A

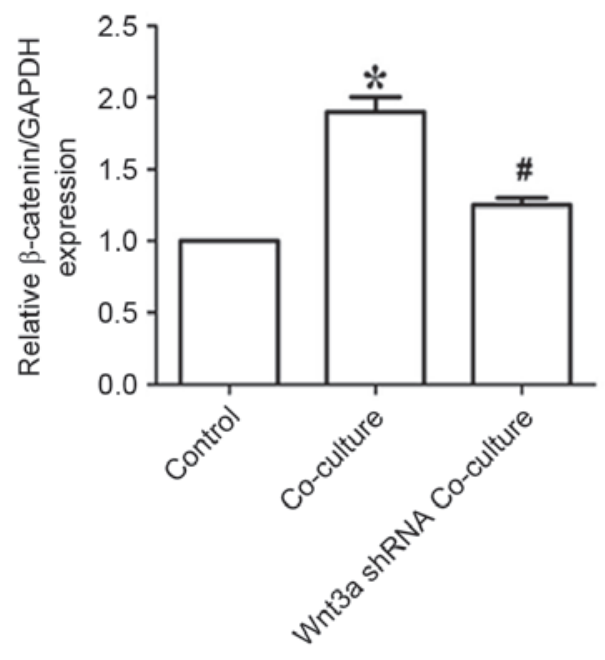

D
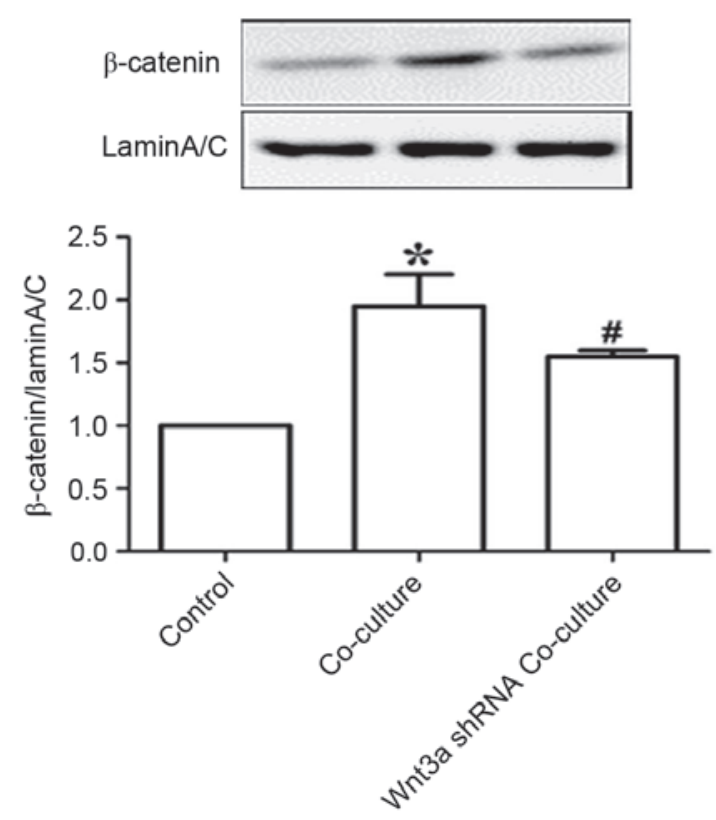

B
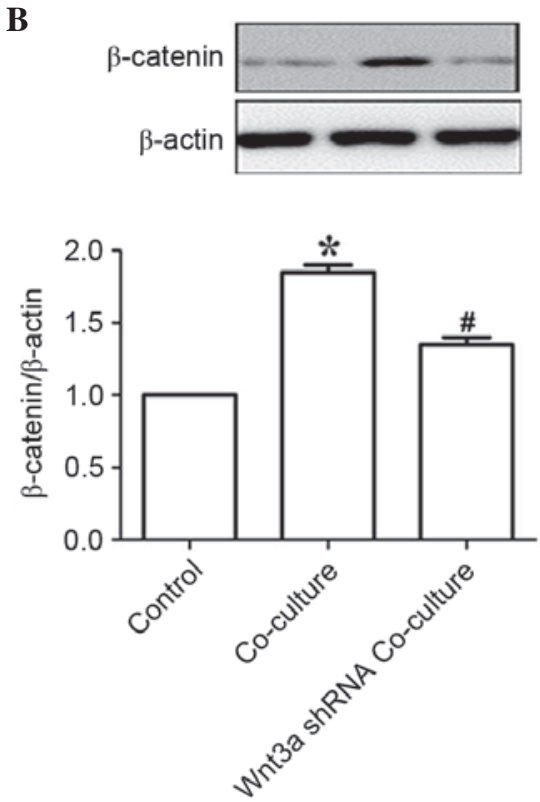

C
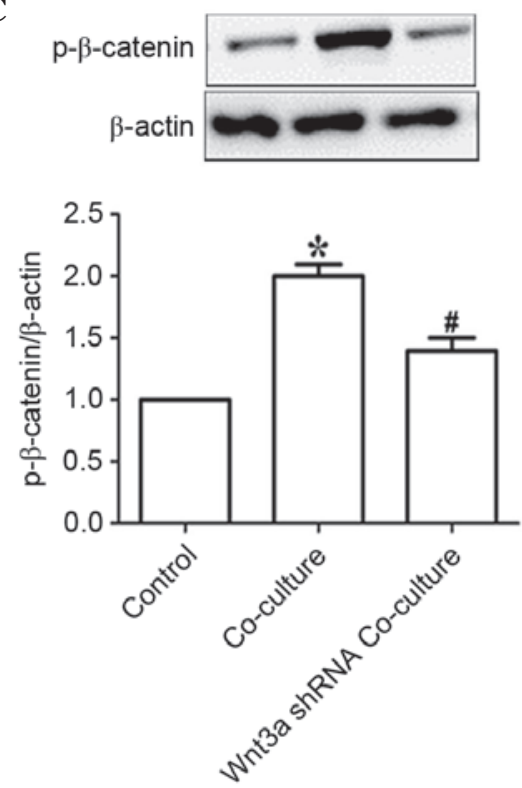

$\mathbf{E}$
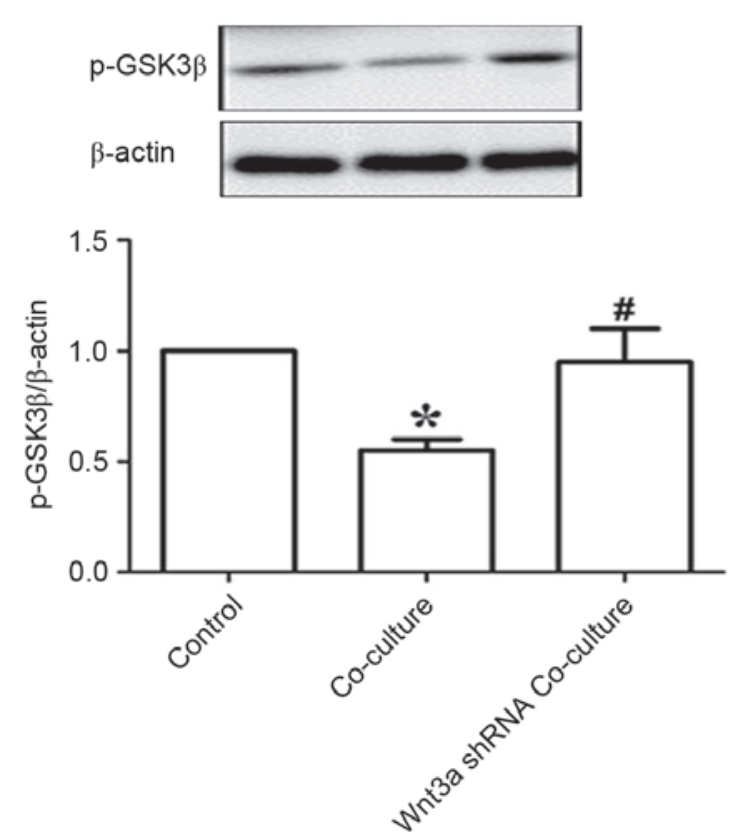

Figure 5. Wnt3a knockdown inhibits $\beta$-catenin signaling activation in the co-culture system. (A) Following co-culture with EPCs for 7 days, reverse transcription-quantitative polymerase chain reaction analysis was used to determine the mRNA levels of $\beta$-catenin. GAPDH was used as the housekeeping gene. Western blot analysis was used to determine the protein levels of (B) $\beta$-catenin, (C) p- $\beta$-catenin, (D) nuclear $\beta$-catenin and (E) p-GSK-3 $\beta$, $\beta$-actin was used as a loading control. Values are expressed as the mean \pm standard error of the mean ( $\mathrm{n}=3$ ). ${ }^{*} \mathrm{P}<0.05$, vs. NSCs alone; ${ }^{\#} \mathrm{P}<0.05$, vs. EPC co-culture. EPCs, endothelial progenitor cells; NSCs, neural stem cells; GSK3 $\beta$, glycogen synthase kinase $3 \beta$; p-, phosphorylated; shRNA, short hairpin RNA.

pathway reduces myelination and neurogenesis, which leads to cognitive dysfunction in rats (17). Therefore, to elucidate the mechanisms by which co-culture with EPCs promotes NSC proliferation and differentiation, the present study investigated the signaling pathway of the co-culture system. Several findings confirmed that the activation of Wnt3a/ $\beta$-catenin signaling by co-culture with EPCs was critical for EPC-mediated NSC proliferation and differentiation: i) Co-culture with EPCs markedly promoted NSC proliferation, the number of neurospheres and NSC differentiation, which was accompanied by increased mRNA and protein expression levels of $\beta$-catenin and Wnt3a; ii) Wnt3a knockdown in the EPCs decreased the proliferation of NSCs, the number of neurospheres and the differentiation of NSCs in the co-culture system. Wnt3a knockdown in the EPCs eliminated the EPCs-mediated increase in the mRNA and protein expression levels of $\beta$-catenin and Wnt3a, and reversed the EPC-mediated phosphorylation and nuclear translocation of $\beta$-catenin, which was in accordance with a previous report (35); iii) co-culture with EPCs reduced the phosphorylation of GSK-3 $\beta$ in the NSCs, and Wnt3a knockdown in the EPCs significantly increased the phosphorylation of GSK-3 $\beta$ 
in the co-culture system. GSK-3 $\beta$, a multifunctional protein kinase, acts as a key and negative regulator of the classical Wnt/ $\beta$-catenin signaling pathway, and is responsible for the phosphorylation and downregulation of $\beta$-catenin $(36,37)$. Taken together, the results of the present study demonstrated that Wnt3a/ $\beta$-catenin was critical in EPC-mediated NSC proliferation and differentiation.

In conclusion, the results of the present study suggested that co-culture with EPCs promoted the proliferation and differentiation of NSCs through modulation of the Wnt3a/ $\beta$-catenin and GSK-3 $\beta$ signaling pathway. The present study provided molecular insight into the EPC-mediated effects of neurogenesis during the process of repair following spinal cord injury.

\section{Acknowledgements}

This study was supported by grants from the National Natural Science Foundation of China (grant no 81171173) andt the Anhui Provincial Natural Science Foundation (grant no 11040606Q25).

\section{References}

1. La Spada A and Ranum LP: Molecular genetic advances in neurological disease: Special review issue. Hum Mol Genet 19: R1-R3, 2010.

2. Moyse E, Segura S, Liard O, Mahaut S and Mechawar N: Microenvironmental determinants of adult neural stem cell proliferation and lineage commitment in the healthy and injured central nervous system. Curr Stem Cell Res Ther 3: 163-184, 2008

3. Yin Y, Huang P, Han Z, Wei G, Zhou C, Wen J, Su B, Wang X and Wang Y: Collagen nanofibers facilitated presynaptic maturation in differentiated neurons from spinal-cord-derived neural stem cells through MAPK/ERK1/2-Synapsin I signaling pathway. Biomacromolecules 15: 2449-2460, 2014.

4. Ogawa Y, Sawamoto K, Miyata T, Miyao S, Watanabe M, Nakamura M, Bregman BS, Koike M, Uchiyama Y, Toyama Y and Okano H: Transplantation of in vitro-expanded fetal neural progenitor cells results in neurogenesis and functional recovery after spinal cord contusion injury in adult rats. J Neurosci Res 69: 925-933, 2002.

5. Brustle O and McKay RD: Neuronal progenitors as tools for cell replacement in the nervous system. Curr Opin Neurobiol 6 : 688-695, 1996.

6. Qu K, Wang Z, Lin XL, Zhang K, He XL and Zhang $\mathrm{H}$ : MicroRNAs: Key regulators of endothelial progenitor cell functions. Clin Chim Acta 448: 65-73, 2015.

7. Zampetaki A, Kirton JP and Xu Q: Vascular repair by endothelial progenitor cells. Cardiovasc Res 78: 413-421, 2008.

8. Paczkowska E, Rogińska D, Pius-Sadowska E, Jurewicz A, Piecyk K, Safranow K, Dziedziejko V, Grzegrzółka R, Bohatyrewicz A and Machaliński B: Evidence for proangiogenic cellular and humoral systemic response in patients with acute onset of spinal cord injury. J Spinal Cord Med 38: 729-744, 2015

9. Ottone C, Krusche B, Whitby A, Clements M, Quadrato G, Pitulescu ME, Adams RH and Parrinello S: Direct cell-cell contact with the vascular niche maintains quiescent neural stem cells. Nat. Cell Biol 16: 1045-1056, 2014.

10. Kamei N, Kwon SM, Ishikawa M, Ii M, Nakanishi K, Yamada K, Hozumi K, Kawamoto A, Ochi M and Asahara T: Endothelial progenitor cells promote astrogliosis following spinal cord injury through Jagged1-dependent Notch signaling. J Neurotrauma 29: 1758-1769, 2012.

11. Chang Y, Jia X, Wei F, Wang C, Sun X, Xu S, Yang X, Zhao Y, Chen J, Wu H, Zhang L and Wei W: CP-25, a novel compound, protects against autoimmune arthritis by modulating immune mediators of inflammation and bone damage. Sci Rep 6: 26239, 2016.
12. Lendahl U,Zimmerman LB and McKay RD: CNS stem cells express a new class of intermediate filament protein. Cell 60: 585-595, 1990.

13. Li Y, Liu L, Barger SW and Griffin WS: Interleukin-1 mediates pathological effects of microglia on tau phosphorylation and on synaptophysin synthesis in cortical neurons through a p38-MAPK pathway. J Neurosci 23: 1605-1611, 2003.

14. Dráberová E, Del Valle L, Gordon J, Marková V, Smejkalová B, Bertrand L, de Chadarévian JP, Agamanolis DP, Legido A, Khalili K, et al: Class III beta-tubulin is constitutively coexpressed with glial fibrillary acidic protein and nestin in midgestational human fetal astrocytes: Implications for phenotypic identity. J Neuropathol Exp Neurol 67: 341-354, 2008.

15. Livak KJ and Schmittgen TD: Analysis of relative gene expression data using real-time quantitative PCR and the 2(-Delta Delta C(T)) Method. Methods 25: 402-408, 2001.

16. Wang L, Liu Y, Li S, Long ZY and Wu YM: Wnt signaling pathway participates in valproic acid-induced neuronal differentiation of neural stem cells. Int J Clin Exp Pathol 8: 578-585, 2015.

17. Tiwari SK, Agarwal S, Chauhan LK, Mishra VN and Chaturvedi RK: Bisphenol-A impairs myelination potential during development in the hippocampus of the rat brain. Mol Neurobiol 51: 1395-1416, 2015.

18. Shintani S, Murohara T, Ikeda H, Ueno T, Sasaki K, Duan J and Imaizumi T: Augmentation of postnatal neovascularization with autologous bone marrow transplantation. Circulation 103: 897-903, 2001.

19. Garlanda C and Dejana E: Heterogeneity of endothelial cells. Specific markers. Arterioscler Thromb Vasc Biol 17: 1193-1202, 1997.

20. Gould TD and Manji HK: The Wnt signaling pathway in bipolar disorder. Neuroscientist 8: 497-511, 2002.

21. Ille F and Sommer L: Wnt signaling: Multiple functions in neural development. Cell Mol Life Sci 62: 1100-1108, 2005.

22. Borrell V and Reillo I: Emerging roles of neural stem cells in cerebral cortex development and evolution. Dev Neurobiol 72: 955-971, 2012.

23. Chojnacki A, Cusulin C and Weiss S: Adult periventricular neural stem cells: Outstanding progress and outstanding issues. Dev Neurobiol 72: 972-989, 2012.

24. Rossi F and Cattaneo E: Opinion: Neural stem cell therapy for neurological diseases: Dreams and reality. Nat Rev Neurosci 3: 401-409, 2002.

25. Kupatt C, Horstkotte J, Vlastos GA, Pfosser A, Lebherz C Semisch M, Thalgott M, Büttner K, Browarzyk C, Mages J, et al: Embryonic endothelial progenitor cells expressing a broad range of proangiogenic and remodeling factors enhance vascularization and tissue recovery in acute and chronic ischemia. FASEB J 19: 1576-1578, 2005.

26. Guo JS, Zeng YS, Li HB, Huang WL, Liu RY, Li XB, Ding Y, Wu LZ and Cai DZ: Cotransplant of neural stem cells and NT-3 gene modified Schwann cells promote the recovery of transected spinal cord injury. Spinal Cord 45: 15-24, 2007.

27. Uchida N, Chen K, Dohse M, Hansen KD, Dean J, Buser JR, Riddle A, Beardsley DJ, Wan Y, Gong X, et al: Human neural stem cells induce functional myelination in mice with severe dysmyelination. Sci Transl Med 4: 155ra136, 2012.

28. Wang G, Ao Q, Gong K, Zuo H, Gong Y and Zhang X: Synergistic effect of neural stem cells and olfactory ensheathing cells on repair of adult rat spinal cord injury. Cell Transplant 19: $1325-1337,2010$.

29. Habisch HJ, Liebau S, Lenk T, Ludolph AC, Brenner R and Storch A: Neuroectodermally converted human mesenchymal stromal cells provide cytoprotective effects on neural stem cells and inhibit their glial differentiation. Cytotherapy 12: 491-504, 2010.

30. Shen Q, Goderie SK, Jin L, Karanth N, Sun Y, Abramova N, Vincent P, Pumiglia K and Temple S: Endothelial cells stimulate self-renewal and expand neurogenesis of neural stem cells. Science 304: 1338-1340, 2004.

31. Daneman R, Zhou L, Agalliu D, Cahoy JD, Kaushal A and Barres BA: The mouse blood-brain barrier transcriptome: A new resource for understanding the development and function of brain endothelial cells. PLoS One 5: e13741, 2010.

32. Imura T, Tane K, Toyoda N and Fushiki S: Endothelial cell-derived bone morphogenetic proteins regulate glial differentiation of cortical progenitors. Eur J Neurosci 27: 1596-1606, 2008.

33. Lai B, Mao XO, Greenberg DA and Jin K: Endothelium-induced proliferation and electrophysiological differentiation of human embryonic stem cell-derived neuronal precursors. Stem Cells Dev 17: 565-572, 2008 . 
34. Tiwari SK, Agarwal S, Tripathi A and Chaturvedi RK: Bisphenol-A mediated inhibition of hippocampal neurogenesis attenuated by curcumin via canonical Wnt pathway. Mol Neurobiol 53: 3010-3029, 2016.

35. Flentke GR, Garic A, Amberger E, Hernandez M and Smith SM: Calcium-mediated repression of beta-catenin and its transcriptional signaling mediates neural crest cell death in an avian model of fetal alcohol syndrome. Birth Defects Res A Clin Mol Teratol 91: 591-602, 2011
36. Willert K and Nusse R: Beta-catenin: A key mediator of Wnt signaling. Curr Opin Genet Dev 8: 95-102, 1998.

37. Khiari M, Arfaoui A, Kriaa L, Chaar I, Amara S, Lounis MA, Sammoud S, Dhraeif M, Gharbi L, Mzabi-Regaya S and Bouraoui S: The prognostic value of the immunohistochemical expression and mutational pattern of the key mediator of Wnt signaling: Beta-catenin in Tunisian patients with colorectal carcinoma. Appl Immunohistochem Mol Morphol 20: 62-70, 2012. 